Universitas, Volumen 1, Año 1, 2007, 14-20

(C) 2007 UNAN-León, Editorial Universitaria

\title{
Metrología Química I: Calibración de un pHmetro y Control de Calidad
}

\author{
Marco Delgado, Manuel Vanegas y Gustavo Delgado*. \\ Laboratorio de Análisis de Trazas de Metales Pesados (LATMP), Departamento de Química, Facultad de Ciencias, Universidad \\ Nacional Autónoma de Nicaragua, León. (UNAN-León).
}

\section{RESUMEN}

\begin{abstract}
El objetivo principal del presente trabajo es establecer una metodología confiable para la calibración de un pHmetro utilizando un calibrador de $\mathrm{pH}$ y soluciones de referencia de $\mathrm{pH}$ certificadas. En primer lugar se verificaron las especificaciones de la calibración del pHmetro declaradas por el fabricante, utilizando un calibrador de $\mathrm{pH}$. Se verificó la linealidad de la respuesta de potencial del electrodo para diferentes $\mathrm{pH}$ y se evaluó la eficiencia del electrodo, encontrándose un valor de $98.39 \%$. Se determinó el modelo de calibración entre la respuesta de pH del instrumento y los valores de $\mathrm{pH}$ establecidos en soluciones de referencia certificadas de $\mathrm{pH} 4.00,7.00$ y 10.00 , encontrándose un valor de pendiente igual a 1 y un intercepto de 0 , lo que indica el buen funcionamiento del instrumento. Por último, se hizo un estudio de la repetibilidad durante 5 días consecutivos utilizando la prueba de Bartlett para la comparación de las precisiones de cada serie de resultados y ANOVA de un factor para la comparación entre las medias de cada día. Por último, se elaboraron las cartas de control para el pH de las soluciones de referencia certificadas, con el fin de llevar un control de calidad en las mediciones de rutina.
\end{abstract}

Palabras claves: Metrología química, calibración de pH-metro, cartas de control en medición de pH, control de calidad en medición de $\mathrm{pH}$.

\section{INTRODUCCIÓN}

Existen muchas aplicaciones en las que se utiliza la técnica potenciométrica para .la medición del pH en diferentes matrices ${ }^{[1]}$. En análisis de agua tiene una amplia aplicación en la determinación de acidez y alcalinidad $^{[2,3]}$. La medición del pH en la fabricación de productos alimenticios es fundamental para la calidad del producto ${ }^{[4]}$. El control del pH en fluidos biológicos es muy importante para el diagnóstico de enfermedades ${ }^{[5]}$. El control de la acidez en los documentos de valor histórico es de primordial importancia para su conservación y preservación ${ }^{[6]}$.

En los procesos de intercambio comercial globalizados se exigen que las mediciones de un parámetro característico del producto deben ser trazables al sistema internacional de unidades ${ }^{[7]}$. Esto significa que todo instrumento de medición analítica debe estar debidamente calibrado antes de ser utilizado en los ensayos de rutina. Así, la medición del pH de una muestra de agua debe ser trazable al sistema internacional de unidades (SI). Para ello, es necesario utilizar soluciones de referencia certificadas ${ }^{[8]}$ trazables a patrones de referencia de laboratorios nacionales de metrología, quienes disponen de patrones de referencia primarios (MRP) trazables al SI. Entre los laboratorios nacionales a nivel del continente americano se destacan el Instituto Nacional de Normas y Tecnología (NIST) en los Estados Unidos y el Centro Nacional de Metrología (CENAM) en México.

\footnotetext{
*Autor para correspondencia: gdelgado@unanleon.edu.ni
}

En el presente trabajo se pretende establecer una metodología para la calibración del sistema potenciométrico en la medición de $\mathrm{pH}$, utilizando un simulador o calibrador de $\mathrm{pH}$ y soluciones de referencia certificadas de $\mathrm{pH} 4.00,7.00$ y 10.00 . Para ello, se hizo una evaluación de la linealidad de la respuesta de potencial en función del $\mathrm{pH}$ y la eficiencia del electrodo. Así mismo, se realizó un estudio de repetibilidad y se elaboraron cartas de control para identificar los cambios del proceso de medición en análisis de rutina.

\section{PRINCIPIO DE LA CALIBRACIÓN}

El circuito básico de un pHmetro o medidor de voltaje se presenta en la figura 1. El funcionamiento se puede describir de la siguiente manera: la celda, constituida por el electrodo indicador y el de referencia, está conectada al amplificador seguidor de voltaje que tiene una alta impedancia $10^{12} \Omega^{[9]}$. La función de este amplificador es amplificar la potencia y tiene grandes corrientes de salida; se le denomina buffer, porque el voltaje Vi permanece inalterable. El diseño del segundo amplificador permite amplificar el voltaje, produciendo un voltaje $\mathrm{V} 0$ de salida mucho mayor que el de entrada Vi. La resistencia R2>R1 y dado que el amplificador es inversor, la ganancia es la razón negativa ${ }^{[9]}$ de $\mathrm{R} 2$ con respecto a R1, es decir $\mathrm{G}=-\mathrm{R} 2 / \mathrm{R} 1$. Por tanto, el voltaje de salida V0 tiene un valor amplificado de Vi en un factor $\mathrm{G}$. 


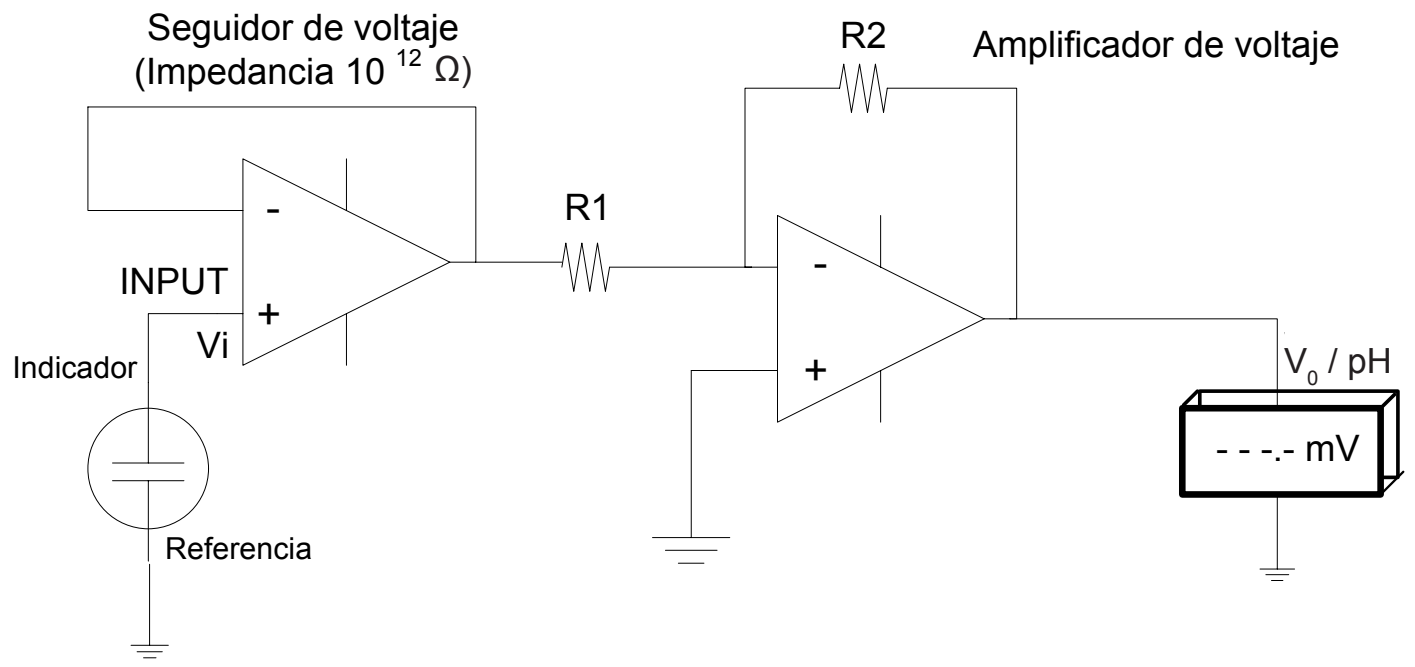

Figura 1. Circuito simplificado de un $\mathrm{pH}$-metro

Todo instrumento de medición debe estar calibrado antes de su utilización ${ }^{[10]}$. Esto significa que es necesario verificar las especificaciones de un $\mathrm{pH}$ metro de acuerdo a lo declarado por el fabricante en el manual del usuario. Existen dos pruebas básicas para verificar las especificaciones [11]: 1) al cortocircuitar la entrada (INPUT) del electrodo indicador y de referencia el valor de $\mathrm{pH}$ debe ser 7 y el potencial $000.0 \mathrm{mV}$; 2) al proporcionar un valor de potencial de $\pm 89 \mathrm{mV}$ en las entradas de la celda con un calibrador, el $\mathrm{pH}$ registrado por el pH-metro debe ser de 5.5 y 8.5 .

El pHmetro está diseñado para registrar un valor de potencial de cero voltios a pH 7. Los electrodos de vidrio y de referencia son también diseñados para obtener el mismo potencial cuando los electrodos se sumergen en una solución buffer de $\mathrm{pH} 7^{[1]}$. El modelo matemático que explica este principio se expresa en la ecuación (1).

$$
E_{v}=-\frac{R T}{F}(1000) \operatorname{Ln}(10)(p H-7)
$$

Donde:

$\mathrm{E}_{\mathrm{v}}$, es el potencial medido en milivoltios, $\mathrm{V}_{0}$.

$\mathrm{R}=8.31441 \mathrm{~J} \mathrm{~mol}^{-1} \mathrm{k}^{-1}$

$\mathrm{F}=96484.56 \mathrm{C} \mathrm{mol}^{-1}$

$\mathrm{T}=\left(273.15+\mathrm{t}^{\circ} \mathrm{C}\right) \mathrm{K}$

De acuerdo a la ecuación anterior, a $25^{\circ} \mathrm{C}$ se tiene una pendiente de $-59.16 \mathrm{mV} / \mathrm{pH}$ y un intercepto de $414.1 \mathrm{mV}$. Por tanto, cuando se calibra el sistema potenciométrico (pHmetro + electrodos) se debe verificar la eficiencia de la respuesta del electrodo comparando el valor de la pendiente obtenida para las lecturas de $\mathrm{pH}$ de diferentes soluciones de referencias certificadas (o tampones trazable a patrones primarios) con respecto al valor teórico a $25^{\circ} \mathrm{C}{ }^{[11]}$.

\section{MATERIALES Y MÉTODOS}

\subsection{Materiales}

Durante el proceso de calibración se utilizaron soluciones de referencia de $\mathrm{pH} 4.00,7.00$ y 10.00 certificadas trazables con NIST, un calibrador de $\mathrm{pH}$ marca HANNA HI931001, un pH-metro TACUSSEL LPH 230T, un baño termostático Vidra FOC BT-9, una unidad refrigerante marca Vidra FOC UR-1, un termómetro digital TESTO 925, electrodos de vidrio combinado con el de platacloruro de plata marca TACUSSEL.

\subsection{Calibración del sistema potenciométrico}

Para calibrar el 0.000 voltios del pH-metro, se cortocircuitaron las entradas correspondientes al del electrodo indicador y al de referencia. Para verificar la calibración en el intervalo de pH 4.00 a 10.00, se conectó el calibrador HANNA y se seleccionaron los $\mathrm{pH} 4.00$, $5.5,7.00,8.5$ y 10.00 y se registraron en el $\mathrm{pH}$-metro los valores de potencial correspondientes en milivoltios a una temperatura ajustada a $25^{\circ} \mathrm{C}$. Una vez verificado el buen funcionamiento, se conectaron los electrodos de vidrio y de referencia, se sumergió en una solución de $\mathrm{pH} 7.00$ termostatizada a $25^{\circ} \mathrm{C}$ con una precisión de $\pm 0.1^{\circ} \mathrm{C}$, y se realizó la lectura en milivoltios.

Luego se introdujeron electrodos en las soluciones de referencias de $\mathrm{pH} 4.00$ y 10.00 y se realizaron las lecturas correspondientes en milivoltios. Se hicieron 5 lecturas en milivoltios para cada valor de $\mathrm{pH}$. Para verificar la calibración se realizaron las lecturas de las soluciones de referencia en el modo $\mathrm{pH}$, se obtuvo el modelo de calibración y se evaluaron la pendiente y el intercepto. 


\subsection{Repetibilidad y cartas de control}

Se realizan lecturas de $\mathrm{pH}$ de las soluciones de referencia certificadas a $25^{\circ} \mathrm{C}$, durante 5 días consecutivos a razón de 5 réplicas por día. Se evaluó la repetibilidad aplicando las pruebas de Bartlett y ANOVA de un factor. Las cartas de control se elaboraron a partir de las varianzas dentro de los días y entre los días.

\section{RESULTADOS Y DISCUSIÓN}

\subsection{Verificación de la Calibración del pH-metro y calibración del electrodo de vidrio}

\subsubsection{Verificación de las especificaciones del fabricante}

El fabricante declara que el potencial debe ser $0.00 \mathrm{mV}$ cuando se selecciona un $\mathrm{pH} 7.00$; y deben registrarse valores de potencial de $\pm 90 \mathrm{mV}$ para una desviación de 1.5 inferior o superior al valor de $\mathrm{pH}$ de referencia (6.50 y 8.50) [11]. Los resultados obtenidos después de realizar la prueba cortocircuitando las entradas de la celda y con el calibrador o simulador de $\mathrm{pH}$ se observan en la tabla №1.

TABLA No 1: Resultados de la verificación de conformidad con el fabricante

\begin{tabular}{|l|l|l|l|}
\hline $\begin{array}{l}\boldsymbol{p H} \\
\text { simulador }\end{array}$ & $\begin{array}{l}\mathbf{E} \boldsymbol{p H m e t r o /} \\
\boldsymbol{m} \boldsymbol{V}\end{array}$ & $\begin{array}{l}\text { Ev teórico/ } \\
\boldsymbol{m} \boldsymbol{V}\end{array}$ & E fabricante \\
\hline 7.00 & 000 & 000.0 & 000 \\
\hline 6.5 & 89 & 88.7 & 90 \\
\hline 8.5 & -89 & -88.7 & -90 \\
\hline
\end{tabular}

De la tabla se deduce que el $\mathrm{pH}$-metro cumple con las especificaciones declaradas en el manual del fabricante. El valor de potencial teórico (calculado de la ecuación 1) se refiere a un instrumento con resolución de $0.1 \mathrm{mV}$. No obstante, el pHmetro utilizado en este estudio tiene una resolución de $1 \mathrm{mV}$. Es importante remarcar que los valores de referencia establecidos por el fabricante no son exactamente iguales a los valores teóricos hay una diferencia de $1 \mathrm{mV}$.

Con el fin de verificar la exactitud de la respuesta de potencial del instrumento en el rango de $\mathrm{pH}$ donde se realizan las calibraciones rutinarias con soluciones de referencia certificadas se realizaron pruebas para $\mathrm{pH}$ simulados de 4.00 y 10.00. Los resultados obtenidos se presentan en la tabla $\mathrm{N}^{\circ} 2$.

TABLA No 2: Potenciales registrados y teóricos para $\mathrm{pH}$ simulados 4.00 y 10.00 a $25^{\circ} \mathrm{C}$.

\begin{tabular}{|l|l|l|}
\hline $\mathbf{p H}$ simulador & E pHmetro/mV & Ev teórico/mV \\
\hline 4 & 177 & 177.5 \\
\hline 10 & -177 & -177.5 \\
\hline
\end{tabular}

Se observa claramente que la respuesta del pHmetro responde a los valores teóricos. La diferencia estriba en la resolución del instrumento de medición, tal a como se dijo anteriormente. El pHmetro utilizado en el experimento tiene una resolución de $1 \mathrm{mV}$ y si se utilizara un instrumento con una resolución de 0.1 $\mathrm{mV}$ se hubiera podido evaluar con mayor precisión la eficiencia de la medición de potencial. Es importante hacer notar que debido a que la respuesta del $\mathrm{pH}$-metro no presentaba variaciones en las réplicas, no se pudo utilizar la estadística clásica para la comparación entre los resultados experimentales y los teóricos. Esto es normal para instrumentos digitales con poca resolución ${ }^{[13]}$.

\subsubsection{Calibración del sistema potencio-métrico (pHmetro + electrodos)}

El proceso de calibración del sistema, utilizando un electrodo combinado de vidrio, se efectuó a través de 3 soluciones de referencia certificadas $(\mathrm{pH} 4.00,7.00$ y 10.00). Los resultados se presentan en la tabla №3.

TABLA No 3: Valores de potencial registrados para soluciones de referencia de $\mathrm{pH}$ 4.00, 7.00 y 10.0.

\begin{tabular}{|l|l|l|l|l|l|l|l|}
\hline $\boldsymbol{p H}$ & $\mathbf{E 1}$ & $\mathbf{E 2}$ & $\mathbf{E 3}$ & $\mathbf{E 4}$ & $\mathbf{E 5}$ & $\boldsymbol{E}$ prom & $\boldsymbol{S}^{\mathbf{2}}$ \\
\hline 4.00 & 166 & 168 & 168 & 168 & 167 & 167.75 & 0.894 \\
\hline 7.00 & -7 & -6 & -6 & -7 & -6 & -6.25 & 0.300 \\
\hline 10.00 & -182 & -178 & -183 & -183 & -182 & -181.5 & 4.3 \\
\hline
\end{tabular}

El análisis de regresión lineal ponderado por el método de los mínimos cuadrados ${ }^{[12]}$ arroja los resultados que se muestran en la Tabla No 4.

TABLA No 4: Resultados del análisis de regresión lineal a $25^{\circ} \mathrm{C}$.

\begin{tabular}{|c|c|c|c|c|c|}
\hline $\begin{array}{c}\text { Pendiente } \\
\left(\mathbf{b}_{1}\right)\end{array}$ & $\mathbf{S b}_{1}$ & $\begin{array}{c}\text { Intercepto } \\
\left(\boldsymbol{b}_{\boldsymbol{\alpha}}\right)\end{array}$ & $\boldsymbol{S b}_{\circ}$ & $\begin{array}{c}\boldsymbol{S}^{2}{ }_{\mathrm{x} / \mathrm{y}} \text { Var. } \\
\text { residual }\end{array}$ & $\boldsymbol{r}^{2}$ \\
\hline-58.208 & 0.15146 & 400 & 1.0007 & 0.155 & 0.9999 \\
\hline
\end{tabular}

Se puede observar una buena linealidad, $99.99 \%$ en el coeficiente de determinación $r^{2}$. Sin embargo, la pendiente aparentemente difiere del valor teórico (-59.16 obtenido de la ecuación 1). Si se asume la hipótesis de que la pendiente experimental debe ser igual a la teórica, es necesario verificar esta hipótesis aplicando la prueba $Z^{[14]}$ :

$$
-1.96<Z_{c}=\frac{b_{1}-\beta_{1}}{S_{b 1}}<1.96
$$

Donde $\boldsymbol{b}_{1}=-58.208, \boldsymbol{S}_{\boldsymbol{b} 1}=0.15146$ y $\boldsymbol{\beta}_{1}=-59.16$ 
El valor de $z_{c}$ es de 6.286, está fuera del intervalo. Por lo que se puede afirmar que existe una diferencia significativa entre la pendiente teórica y la experimental. Esto significa que la eficiencia del electrodo no es $100 \%$ y se puede calcular de acuerdo a la siguiente expresión a $25^{\circ} \mathrm{C}$ :

$$
P_{25} \%=\frac{b_{1}}{\beta_{1}} 100
$$

La eficiencia del electrodo es $98.39 \%$. Si tomamos en cuenta que la corrección de la pendiente que se puede efectuar en los pHmetros oscila entre el 80 al $105 \%{ }^{[1]}$, dependiendo del fabricante, podemos afirmar que el electrodo tiene muy buena respuesta, casi el $100 \%$ de eficiencia.

\subsubsection{Verificación de la calibración}

Con el objeto de verificar la calibración del sistema potenciométrico, se efectuaron lecturas repetidas de $\mathrm{pH}$ para las soluciones de referencia de $\mathrm{pH} 4.00,7.00$ y 10.00. Los resultados experimentales y el análisis de regresión lineal se presentan en la Tabla No 5 y 6 respectivamente.

TABLA N $N^{\circ}$ : Lecturas de $\mathrm{pH}$ a diferentes buffers, promedio y varianzas

\begin{tabular}{|l|l|l|l|l|l|l|l|}
\hline \multicolumn{1}{|c|}{ Buffers } & \multicolumn{1}{c|}{$\mathbf{p H}_{\mathbf{1}}$} & \multicolumn{1}{c|}{$\mathbf{p H}_{\mathbf{2}}$} & \multicolumn{1}{c|}{$\mathbf{p H}_{\mathbf{3}}$} & \multicolumn{1}{c|}{$\mathbf{p H}_{\mathbf{4}}$} & \multicolumn{1}{c|}{$\mathbf{p H}_{\mathbf{5}}$} & \multicolumn{1}{c|}{$\mathbf{p} \overline{\boldsymbol{H}}$} & \multicolumn{1}{c|}{$\mathbf{S}^{2} \boldsymbol{p H}$} \\
\hline 4.00 & 4.03 & 4.01 & 4.02 & 4.03 & 4.03 & 4.02 & $8.0 E-5$ \\
\hline 7.00 & 7.00 & 7.01 & 7.00 & 6.99 & 7.00 & 7.00 & $5.0 E-5$ \\
\hline 10.00 & 9.98 & 9.99 & 10.08 & 10.06 & 10.05 & 10.03 & $1.2 E-3$ \\
\hline
\end{tabular}

TABLA No 6: Resultados del análisis de regresión lineal ponderado.

\begin{tabular}{|l|l|}
\hline Parámetro & Valores \\
\hline$b_{0}$ & $3.51 E-2$ \\
\hline$b_{1}$ & 0.9955 \\
\hline$S b_{o}$ & 0.0286 \\
\hline$S b_{1}$ & 0.0046 \\
\hline$r^{2}$ & 0.9999 \\
\hline$S_{X / Y}{ }^{2}$ & $1.6 E-4$ \\
\hline
\end{tabular}

Se considera que la calibración es excelente si se cumple la siguiente condición: $b_{0}=\beta_{0}=0$ y $b_{1}=\beta_{1}=1$

Para verificar esta hipótesis, se aplicó la prueba z:

$$
-1.96<z_{C}=\frac{b_{i}-\beta_{i}}{S b_{i}}<1.96
$$

Donde $i=0,1$ indicando el intercepto y la pendiente.

Si se toman los datos de la tabla $N^{\circ} 6$ y se sustituyen en la ecuación (4), se tienen valores de $Z_{c} 1.23$ para el intercepto y 0.97 para la pendiente. En ambos casos se cumple que $-1.96<Z<1.96$, lo que indica efectivamente que el intercepto es 0 y la pendiente es 1 , y se puede afirmar que la calibración es excelente.

A pesar que el número de puntos de la recta de calibración es solamente 3 , existe muy buena precisión en el modelo de calibración. Esto se verifica con el valor de RSD $\left(=100 \mathrm{~S}_{x / y} / p \bar{H}\right)$ del modelo. Este valor es solamente de $0.18 \%$, tomando como referencia un valor menor al $1 \%$.

\subsection{Evaluación de la Repetibilidad}

Para comprobar que los resultados de las mediciones son repetibles a través del tiempo, se procedió a medir el $\mathrm{pH}$ de tres soluciones buffer $(4.00,7.00,10.0)$ a $25^{\circ} \mathrm{C}$ durante 5 días consecutivos, realizando 5 réplicas de la lectura por cada día. Los resultados se pueden observar en la tabla No 7.

Para verificar si hay buena repetibilidad en las 5 series de resultados, se compararon las varianzas a través de la prueba de Bartlett ${ }^{[14]}$. Los resultados de esta prueba estadística se presentan en la tabla $\mathrm{N}^{\circ} 8$. En todos los casos se observa que el valor del parámetro de Bartlett $M$, es menor que la chi-cuadrada $\chi^{2}$ de las tablas estadísticas para $n_{i}-1$ grados de libertad y un $95 \%$ de confianza, donde ni es el número de réplicas por día.

Con el fin de verificar la igualdad de las medias en los 5 días, se aplicó la prueba ANOVA (análisis de varianza) de un factor y se calcularon las varianzas de repetibilidad $\left(\mathrm{S}_{\mathrm{r}}^{2}\right)$ y la de la precisión intermedia o entre días $\left(\mathrm{S}_{\mathrm{ED}}^{2}\right)$ [15] (estas dos varianzas fueron utilizadas para elaborar las cartas de control). Los resultados de esta prueba se presentan en la tabla $N^{\circ} 9$, donde se demuestra que no hay diferencias significativas entre las medias. Todos los valores de $F_{c}$ son menores que los de las tablas estadísticas para un nivel de confianza del $95 \%$.

La repetibilidad en las mediciones de $\mathrm{pH}$ para los 3 valores de referencia se calcula utilizando la desviación estándar relativa expresada como porcentaje (ecuación 5). Las varianzas residuales representan la repetibilidad de las mediciones de $\mathrm{pH}$. 
TABLA N $N^{\circ}$ : Valores de $\mathrm{pH}$ registrados en el $\mathrm{pH}$-metro para las soluciones de referencia de $\mathrm{pH} 4.00,7.00$ y 10.00 a $25^{\circ} \mathrm{C}$.

\begin{tabular}{|l|l|l|l|l|l|}
\hline $\mathbf{p} \boldsymbol{H}_{\mathbf{B}}$ & Dia 1 & Dia 2 & Dia 3 & Dia 4 & Dia 5 \\
\hline \multirow{5}{*}{4.01} & 4.01 & 4.02 & 4.00 & 4.00 & 4.00 \\
\cline { 2 - 6 } & 4.00 & 4.02 & 4.00 & 4.01 & 4.00 \\
\cline { 2 - 6 } & 3.98 & 4.00 & 4.00 & 4.00 & 3.98 \\
\hline $\bar{x}_{j}$ & $\mathbf{4 . 0 0 2}$ & $\mathbf{4 . 0 1}$ & $\mathbf{3 . 9 9 6}$ & $\mathbf{4 . 0 0 4}$ & 3.992 \\
\hline varianza & $\mathbf{0 . 0 0 0 2}$ & $\mathbf{0 . 0 0 0 2}$ & $\mathbf{8 E}-05$ & $\mathbf{3 E}-05$ & $\mathbf{0 . 0 0 0 1}$ \\
\hline \multirow{5}{*}{7.00} & 7.02 & 7.02 & 7.00 & 7.01 & 7.00 \\
\cline { 2 - 6 } & 7.02 & 7.02 & 7.00 & 7.00 & 7.00 \\
\cline { 2 - 6 } & 7.02 & 7.01 & 6.97 & 7.00 & 7.01 \\
\cline { 2 - 6 } & 7.02 & 7.01 & 7.00 & 7.00 & 6.98 \\
\cline { 2 - 6 } & 6.97 & 7.01 & 7.00 & 7.05 & 7.00 \\
\hline $\bar{x}_{j}$ & 7.010 & 7.014 & 6.994 & 7.012 & 6.998 \\
\hline varianza & $\mathbf{0 . 0 0 0 5}$ & $\mathbf{3 . 0 \mathrm { E } - 5}$ & $\mathbf{0 . 0 0 0 2}$ & $\mathbf{0 . 0 0 0 5}$ & $\mathbf{0 . 0 0 0 1}$ \\
\hline \multirow{5}{*}{10.00} & 10.05 & 10.1 & 10.05 & 10.03 & 10.05 \\
\cline { 2 - 6 } & 10.0 & 10.01 & 10.00 & 10.03 & 10.03 \\
\cline { 2 - 6 } & 10.04 & 10.0 & 10.05 & 10.05 & 10.00 \\
\cline { 2 - 6 } & 10.03 & 10.05 & 10.01 & 10.05 & 10.03 \\
\cline { 2 - 6 } & 10.05 & 10.01 & 10.05 & 10.01 & 10.05 \\
\hline $\bar{x}_{j}$ & 10.034 & 10.034 & 10.032 & 10.034 & $\mathbf{1 0 . 0 3 2}$ \\
\hline varianza & $\mathbf{0 . 0 0 0 4}$ & $\mathbf{0 . 0 0 1 7}$ & $\mathbf{0 . 0 0 0 6}$ & $\mathbf{0 . 0 0 0 3}$ & $\mathbf{0 . 0 0 0 4}$ \\
\hline
\end{tabular}

TABLA No 8: Resultados del test de Bartlett al 95\% de nivel de confianza.

\begin{tabular}{|l|l|l|}
\hline $\boldsymbol{p H}$ & $\boldsymbol{M}$ & $\chi^{2}$ \\
\hline 4.00 & 9.19 & 9.49 \\
\hline 7.00 & 8.86 & 9.49 \\
\hline 10.00 & 5.81 & 9.49 \\
\hline
\end{tabular}

TABLA No 9: Resultados de la prueba ANOVA de un factor

\begin{tabular}{|c|c|c|c|c|}
\hline$p H$ & $\begin{array}{l}\text { Fuente de } \\
\text { variación }\end{array}$ & Varianzas $\mathrm{Si}^{2}$ & $F c$ & $\boldsymbol{F}_{0.95}$ \\
\hline \multirow{2}{*}{4.00} & Entre días(ED) & 0.00025 & \multirow{2}{*}{2.05} & \multirow{2}{*}{2.87} \\
\hline & Residual(r) & 0.00012 & & \\
\hline \multirow{2}{*}{7.00} & Entre dias(ED) & 0.00040 & \multirow{2}{*}{1.55} & \multirow{2}{*}{2.87} \\
\hline & Residual(r) & 0.00026 & & \\
\hline \multirow{2}{*}{10.00} & Entre dias(ED) & 0.00073 & \multirow{2}{*}{1.47} & \multirow{2}{*}{2.87} \\
\hline & Residual(r) & 0.000494 & & \\
\hline
\end{tabular}

Donde $\mathrm{S}_{\mathrm{r}}^{2}$ es la varianza de repetibilidad y es la media global de los resultados obtenida a partir de la tabla $\mathrm{N}^{\circ}$ 7. Los valores de RSD\% para las lecturas de $\mathrm{pH}$ de las soluciones de referencia se pueden ver en la tabla $\mathrm{N}^{\circ}$ 10. En todos los casos hay una excelente precisión, si tomamos como referencia un valor menor que el $1 \%$.

$$
R S D \%=\frac{\sqrt{S_{r}^{2}}}{\bar{X}} * 100
$$

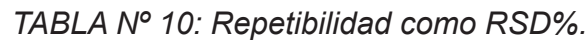

\begin{tabular}{|c|c|}
\hline $\mathbf{p H}$ & $\mathbf{R S D} \%$ \\
\hline 4.00 & 0.3 \\
\hline 7.00 & 0.2 \\
\hline 10.00 & 0.2 \\
\hline
\end{tabular}

\subsection{Cartas de control}

Con el fin de identificar los cambios del proceso de medición en el tiempo, es decir llevar un control de calidad en las mediciones de rutina, se han elaborado las cartas de control para cada valor de $\mathrm{pH}$ medido en las tres soluciones de referencia. La carta de control utilizada en este estudio es la del promedio y la desviación estándar ( $\bar{X}$ y $s$ ). A continuación se detalla la construcción de estos gráficos.

Los límites de control para las medias son calculados considerando errores estándares de $\pm 3 s$. Estos límites son calculados utilizando los factores de control 3-sigma ${ }^{[16,18]}$ según las siguientes fórmulas:

$$
\begin{aligned}
& \mathrm{LC}=\bar{X} \\
& \mathrm{LS}=\mathrm{LC}+\mathrm{A}_{3} s \\
& \mathrm{LI}=\mathrm{LC}-\mathrm{A}_{3} s
\end{aligned}
$$


Los límites de control para la desviación estándar se calculan con las siguientes ecuaciones:

$$
\begin{aligned}
& L C=s \\
& L S=B_{4} s \\
& L I=B_{3} s
\end{aligned}
$$

Donde:

$$
\begin{gathered}
\bar{X}=\frac{1}{p} \sum_{j=1}^{p} \bar{x}_{j} \\
\text { es la medida estándar global de los } \mathrm{p}=5 \text { días } \\
s=\sqrt{S_{E D}^{2}+S_{r}^{2}} \text { desviación estándar global, valores }
\end{gathered}
$$
tomados de la tabla $\mathrm{N}^{\circ} 9$.

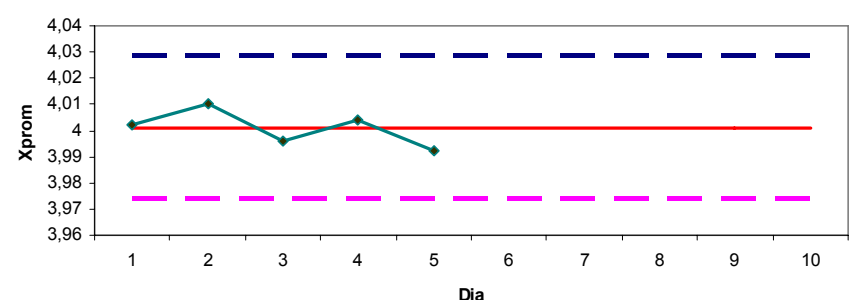

$A_{3}=1.427, B_{3}=0$ y $B_{4}=2.089$ son los factores 3-sigma para 5 réplicas por día encontrados en tablas de Handbook sobre control de calidad para la elaboración de las cartas de control ${ }^{[16,18]}$.

En las figuras 2, 3 y 4 se presentan los gráficos de control para los promedios y las desviaciones estándares de las tres soluciones de referencia de pH 4.00, 7.00 y 10.00 .

En las figuras 2, 3 y 4 se presentan los gráficos de control para los promedios y las desviaciones estándares de las tres soluciones de referencia de pH 4.00, 7.00 y 10.00 .

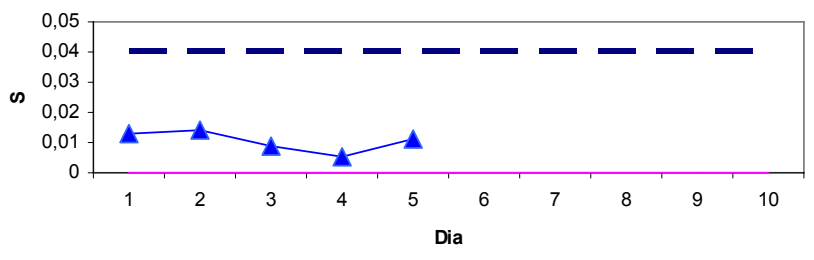

Figura 2. Cartas de control para los promedios y la desviación estándar en la medición de una solución de referencia de pH 4.00.

Los límites de control establecidos son los máximos permisibles para 3-sigma. Para el control de calidad de las mediciones de $\mathrm{pH}$ se deben obtener al menos dos lecturas para cada solución de referencia y ubicarlos en los gráficos: Si algún valor sale fuera de los límites se debe investigar el origen de la anomalía.
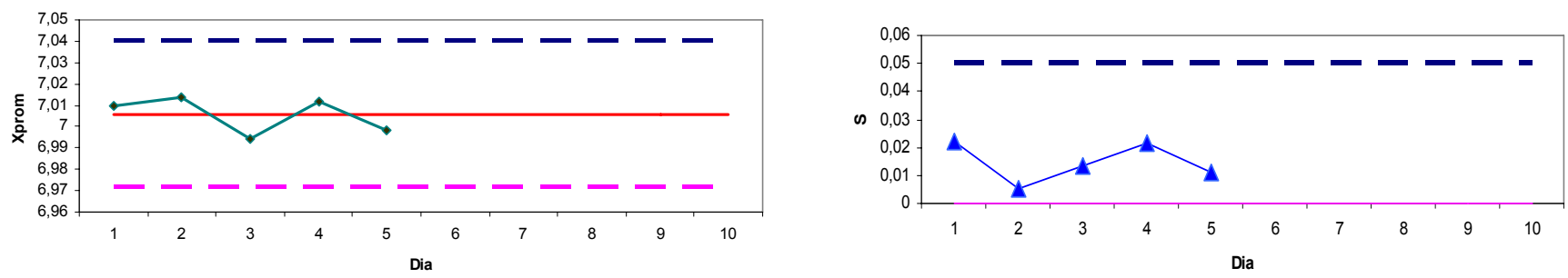

Figura 3. Cartas de control para los promedios y la desviación estándar en la medición de una solución de referencia de $\mathrm{pH} 7.00$.
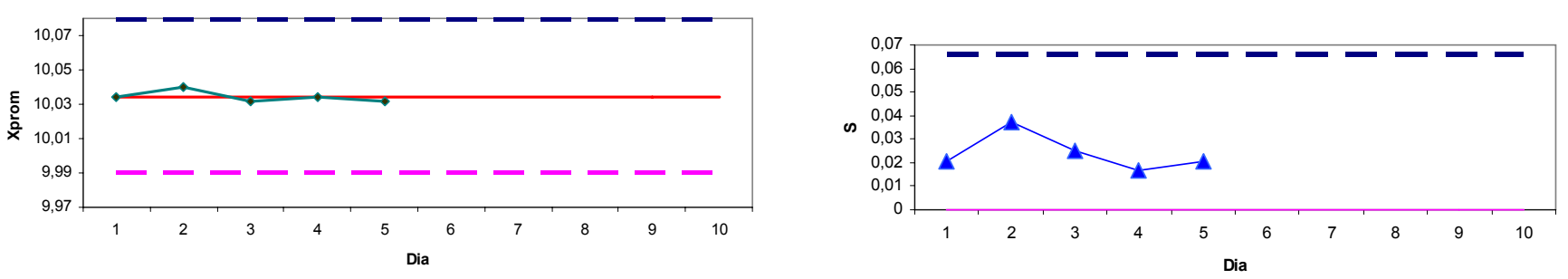

Figura 4. Cartas de control para los promedios y la desviación estándar en la medición de una solución de referencia de $\mathrm{pH} 10.00$. 
Existen otros tipos de cartas de control ${ }^{[16-20]}$ entre los cuales están la de promedio y rango, y las llamadas de CUSUM, por sus siglas en inglés, o sumas acumulativas. Esta última es más sensible para ver las tendencias e identificar más fácilmente una anomalía. El detalle de la construcción de estas cartas se encuentra en la literatura sobre control de calidad ${ }^{[16,19,21]}$.

\section{CONCLUSIONES}

En este estudio se establece una metodología rigurosa para calibrar el pH-metro y el electrodo de vidrio, utilizando un simulador analógico y soluciones de referencias certificadas de tres $\mathrm{pH}$ diferentes $(4.00$, 7.00 y 10.00). Así mismo, se propone el diseño para el estudio de la repetibilidad y la precisión intermedia, aplicando la prueba de Bartlett y la de ANOVA de un factor. Las varianzas fueron utilizadas para elaborar las cartas de control con el fin de establecer el mecanismo para el control del proceso de medición del pH durante los análisis de rutina.

\section{BILIOGRAFÍA}

1. Westcott, C., (1978), pH Measurements, N.Y, Academic Press, p.172.

2. Clescerl, L., Greenberg, A. y Eaton, A., (1998), Standard Methods for the Examination of Water and Wastewater, N.Y., American Publishing Health Association, 20th edition, p. 2-24, 2-26.

3. Midgley, D. and Torrance, K., (1978), Potentiometric Water Analysis, N.Y., John Wiley, p. 135, 147.

4. Ciganda, L. M, Electrodos para medir pH, XIII Seminario de Ing. Biomédica; (2004) Junio, Universidad de la República Oriental de Uruguay, Facultad de Ciencias Médicas e Ingeniería, Montevideo, Uruguay.

5. Alarcon, A. L., Importancia de la medición de pH en suelo, [En Línea]; [Fecha de acceso 29 de marzo del 2007]; URL disponible en: http://mujer.terra.es/ muj/articulo/html/mu24700.htm

6. Edmonson, R., (2002), Directrices para la salvaguardia del patrimonio documental, División de la sociedad de la información, UNESCO, Febrero, Edición Revisada.

7. Eurachem/Citac Guide, (2003), Treceability in Chemical Measurement, UK, Guía de Referencia, 1ra. Edición.
8. EA-04/14, (2003), "The Selection and Use of Reference Materials", Guía de Referencia, European Cooperation for Acreditation.

9. Skoog, D. A., Holler, F. J. y Nieman, T. A., (2005), Análisis Instrumental, Madrid, McGraw Hill, 5ta. Edición, p.63.

10. ISO/IEC 17025, (2005), "Requisitos Generales para la Competencia de los Laboratorios de Ensayo y de Calibración", Norma Internacional, Ginebra, Segunda Edición.

11. Tacussel, (1993), Instrucción Manual, Villeurbanne, Francia, Radiometer Analytical S.A.

12. Mathias, O., (1999), Chemometrics, Weinhein, Alemania, Wiley-VCH, p. 189.

13. Kimothi, S.K., (2000), The Uncertainty of Measurements, Wisconsin, USA, ASQ Quality Press, p. 59.

14. Souvay, P., (2002), Savoir Utiliser la Statistique, Paris, AFNOR, p 182.

15. Kutathmarmmakul, S., Massart, D. L.y SmeyersVerbeke, J., (1999), "Comparison of alternative measurement methods", Analytica Chimica Acta, 391, 203-225.

16. Zimmerman, Steven M., y Icenogle, Marjorie L., (2003), Statistical Quality Control Using Excel, USA, ASQ Quality, 2da. Edición, Press, p. 210, $315,416$.

17. Mullins, E., (1999), "Getting more for your laboratory control charts”, Analyst, 124, 433-442.

18. Juran, J.M., (1988), Juran's Quality Control Handbook, 4ta. Edition, pag. All.3.

19. Kateman, G. , Buydens, L., (1993), Quality Control in Analytical Chemistry, N.Y., John Wiley, p. 28.

20. Ishikawa, K., (1985), Guía de Control de Calidad, USA, UNIPUB, p. 59.

21. Wodsworth, H. M., Stephens, K. y Godfrey, A. B., (2005), Métodos de Control de Calidad, México, Compañía Editorial Continental, 1ra. Edición, p. 258. 